THE JOURNAL OF CHEMICAL PHYSICS 124, 204506 (2006)

\title{
Solvation pressure in chloroform
}

\author{
H. Hubel \\ Physics Department, Queen Mary, University of London, London E1 4NS, United Kingdom \\ D. A. Faux ${ }^{\text {a) }}$ \\ Advanced Technology Institute, University of Surrey, Guildford, Surrey GU2 7XH, United Kingdom
}

R. B. Jones and D. J. Dunstan

Physics Department, Queen Mary, University of London, London E1 4NS, United Kingdom

(Received 10 August 2005; accepted 3 April 2006; published online 25 May 2006)

\begin{abstract}
Molecular dynamics (MD) simulations of chloroform vapor and liquid at normal temperature and pressure and liquid under hydrostatic pressure are presented, giving bond lengths and vibrational frequencies as functions of pressure. The change in bond lengths between vapor and liquid at normal temperature and pressure is consistent with a pressure equivalent to the cohesive energy density (CED) of the liquid, supporting the solvation pressure model which predicts that solvated molecules or nanoparticles experience a pressure equal to the CED of the liquid. Experimental data for certain Raman frequencies of chloroform in the vapor phase, in the liquid, and in the liquid under pressure are presented and compared to $\mathrm{MD}$. Results for $\mathrm{C}-\mathrm{Cl}$ vibrational modes are in general agreement with the solvation pressure model whereas frequencies associated with the $\mathrm{C}-\mathrm{H}$ bond are not. The results demonstrate that masking interactions exist in the real liquid that can be reduced or eliminated in simplified simulations. (C) 2006 American Institute of Physics.
\end{abstract}

[DOI: 10.1063/1.2199531]

\section{INTRODUCTION}

Two of the oldest problems in molecular spectroscopy are the shift in infrared absorption bands from the vapor to the liquid when a molecule is solvated ${ }^{1}$ and the concomitant change in interatomic bond lengths. ${ }^{2}$ These are usually blueshifts of the vibrational frequency indicating bond compression but sometimes redshifts indicating bond stretching. Theoretical models have focused on the properties of the solute molecule and how it is affected by solvent-solute interactions since the early model of Onsager ${ }^{3}$ and Bauer and Magat ${ }^{4}$ who proposed that the dielectric constant of the solvent affects the solute. Following Buckingham et al., ${ }^{5}$ Schweizer and Chandler $^{6}$ developed a statistical mechanical theory invoking competition between short-range and long-range solvent-solute interactions, with a hard-sphere model for the short-range interactions. This theory has been developed further by many authors. ${ }^{2,7}$ Good agreement with experiment has often been found, but with sensitive dependence on fitting parameters such as the hard-sphere radius of the solvent and the length scales of attractive solvent-solute interactions. ${ }^{7}$ These theoretical approaches neglect solventsolvent interactions except indirectly as they determine, for example, the density of the solvent.

In this paper, we adopt a complementary approach to understanding the way vibrational frequencies associated with molecular bonds change when the molecules are solvated. Instead of decomposing the often complex solventsolute interactions into bond-lengthening and bondshortening contributions, it is shown that experimental

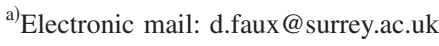

results can also be interpreted in terms of a general solvation pressure added to atom-specific masking interactions. It is shown that for certain vibrational frequencies, the solvation pressure is sufficient to describe quantitatively the change in vibrational frequency associated with the bond shortening of molecular bonds from the vapor to the liquid without recourse to masking interactions. However, atom-specific masking interactions are required to explain the behavior of certain bond types, such as those affected by hydrogen bonding, where the vibrational properties of a bond are especially sensitive to the local environment or where a liquid mixture exhibits nonrandom structure. The nature of the masking interactions may be elucidated by molecular dynamics simulation although it is plausible, also, that the decomposition of the system interactions into solvation pressure plus masking interactions could also be achieved using the statistical mechanical theory of Schweizer and Chandler, ${ }^{6}$ adapted to include solvent-solvent effects.

The solvation pressure is a ubiquitous pressure experienced by all nanoparticles immersed in a solvent and is equal to the cohesive energy density (CED) of the liquid. The CED is defined as the heat of vaporization per unit volume:

$$
\mathrm{CED}=\frac{\Delta U_{\mathrm{vap}}}{V_{L}}
$$

where $\Delta U_{\text {vap }}$ is the internal energy change on vaporization and $V_{L}$ is the volume of the liquid. The CED is also the square of the Hildebrand solubility parameter, which is used in determining the energetics of mixing. ${ }^{8}$ The CED is well known for a range of liquids, for example, 7 kbars for ethanol, 23 kbars for water, and 3.6 kbars for chloroform. ${ }^{9}$ 
The change in bond length due to the solvation pressure depends on the properties of the solvent only. All solutes experience the same solvation pressure and so the solute molecule simply acts as a pressure gauge. In the case of pure liquids, each molecule can be considered to be a solute molecule in a solvent of its own type. Good evidence that solvents exert a pressure on solute molecules and nanoparticles exists for carbon nanotubes, ${ }^{10}$ cosolvent molecules, and starch grains. ${ }^{11}$

The effect of solvation pressure is to shorten the mean length of bonds and hence to increase the Raman frequencies associated with certain bond vibrations. If the solvation pressure was the only influence on a bond then, taking chloroform as an example, the shift in the vibrational frequencies in going from the vapor to the liquid should equal that due to 3.6 kbars of pressure, where $3.6 \mathrm{kbars}$ is the CED for chloroform. In other words, the bonds should shorten from chloroform vapor to liquid by an amount equivalent to $3.6 \mathrm{kbars}$ of hydrostatic pressure applied externally to the liquid. This interpretation will be referred to as the solvation pressure model, or SPM for brevity. The solvation pressure effect must have profound effects in wet chemistry and biochemistry because although solvents of course have specific interactions with solutes, these will be superposed on general effects equivalent to large external positive and negative pressures. ${ }^{12}$

The SPM has been tested previously using molecular dynamics (MD) simulations and, experimentally, using Raman spectroscopy. ${ }^{11,12}$ MD simulations allow the bond lengths of molecules in the liquid or vapor state to be directly compared. Raman spectroscopy allows the effect of a change in bond length to be observed through the change in peak position of certain vibrational modes. Moreover, observation of these changes with the application of hydrostatic pressure allows the magnitude of the vapor-liquid changes to be calibrated with pressure, enabling the ansatz that the solvation pressure is equal to the CED to be tested. For example, MD simulations of ethanol demonstrated that the shortening of most bond lengths in the liquid compared to the vapor was consistent with 7 kbars of hydrostatic pressure, approximately equal in magnitude to the CED. ${ }^{11}$

Quantitative agreement between experiment and the SPM exists for many Raman frequencies and for other effects such as starch gelation, ${ }^{11}$ but there are also discrepancies for many other data. Differences from the simple predictions of the SPM can be attributed to specific solventsolute interactions. ${ }^{11}$ We refer to these as "masking interactions" and these interactions would, of course, be replicated by a fully accurate MD simulation. In ethanol, for example, those bonds affected by the hydrogen-bonding interactions associated with the $\mathrm{OH}$ group revealed the greatest discrepancy from the simple SPM and hence the most significant masking interactions.

In this paper, we present experimental and theoretical data for chloroform and test the solvation pressure plus masking interaction approach to the analysis of the change in vibrational frequencies of a solute. We report a MD study of chloroform vapor, liquid, and liquid under pressure and we compare the results with experimental data on the vapor, on the liquid at ambient pressure, and on the liquid under high pressure in a diamond-anvil cell. MD simulations reproduce the density, bulk modulus, and compressibility of chloroform, demonstrating that the interatomic potentials are of good quality, yet the MD simulations do not reproduce the behavior of certain vibrational modes, suggesting that the generic intramolecular potentials are not sufficiently optimized for chloroform, especially with respect to the $\mathrm{H}$ atom. We argue that the simplifications inherent to the MD model, in particular, the use of pressure-independent potentials, have removed some masking interactions, leaving the ubiquitous effects of solvation pressure clearly revealed. The change in bond lengths between vapor and liquid, however, clearly demonstrates the solvation pressure effect.

The paper is organized as follows. Details of the MD simulations are presented in the following section and the results are analyzed in tandem with experimental results for chloroform in Sec. III where we show that the MD model of chloroform provides good agreement with the predictions of the SPM and that the experimental results produce consistent agreement for some bonds, but not others, particularly with vibrations associated with the $\mathrm{H}$ atom. The conclusions are presented in Sec. IV.

\section{MOLECULAR DYNAMICS SIMULATION}

\section{A. Simulation of liquid chloroform}

We used the MD package DLPOLY $2,{ }^{13}$ as this program is well supported and has been used successfully for a range of simple atomic and molecular systems. For liquid chloroform, the simulation volume is a cube with periodic boundary conditions, initialized with $100 \mathrm{CCl}_{3} \mathrm{H}$ molecules randomly oriented at random positions with each molecule at a sufficient distance from neighboring molecules to ensure that the intermolecular forces are not strong enough to cause molecular breakup during the initial phase of the equilibration. At this stage, the density is about $25 \%$ less than the experimental value for $\mathrm{CCl}_{3} \mathrm{H}$ at $\mathrm{STP}$.

The system energy was minimized by running at zero Kelvin for $5 \times 10^{5}$ time steps of $0.2 \mathrm{fs}$. Then the temperature was raised in steps of $50 \mathrm{~K}$ using successive runs of $2 \times 10^{5}$ time steps as an NVT ensemble (constant number of molecules, volume, and temperature) using the Nosé-Hoover thermostat and equilibrated at $298 \mathrm{~K}$ over a final $1 \times 10^{5}$ time steps. This produces a system of 100 chloroform molecules equilibrated at room temperature but at a density $25 \%$ lower than the experimental value.

The volume was reduced by allowing the simulation cell to shrink during an NPT run (constant number, pressure, and temperature) of $2 \times 10^{4}$ shorter time steps of $0.0002 \mathrm{fs}$. The short time step ensures that the rate of contraction is slow enough to avoid the large intermolecular forces that can lead to the system becoming unstable. The target pressure specified for the $N P T$ ensemble was $30 \mathrm{kbars}$, but this pressure is not reached due to the short duration of the NPT run. Instead, a contraction of the cell by about $0.01 \mathrm{~nm}$ is achieved. The ensemble was then equilibrated at $298 \mathrm{~K}$ as an NVT ensemble over $1 \times 10^{5}$ time steps of $0.2 \mathrm{fs}$. 
TABLE I. The intermolecular and intramolecular Dreiding potentials used in the MD simulations. $E_{0}, K$, and $\varepsilon$ have units of kcal/mol, $r_{0}$ and $\sigma$ have units of $\AA$, and $k$ has units of $\AA^{-1}$.

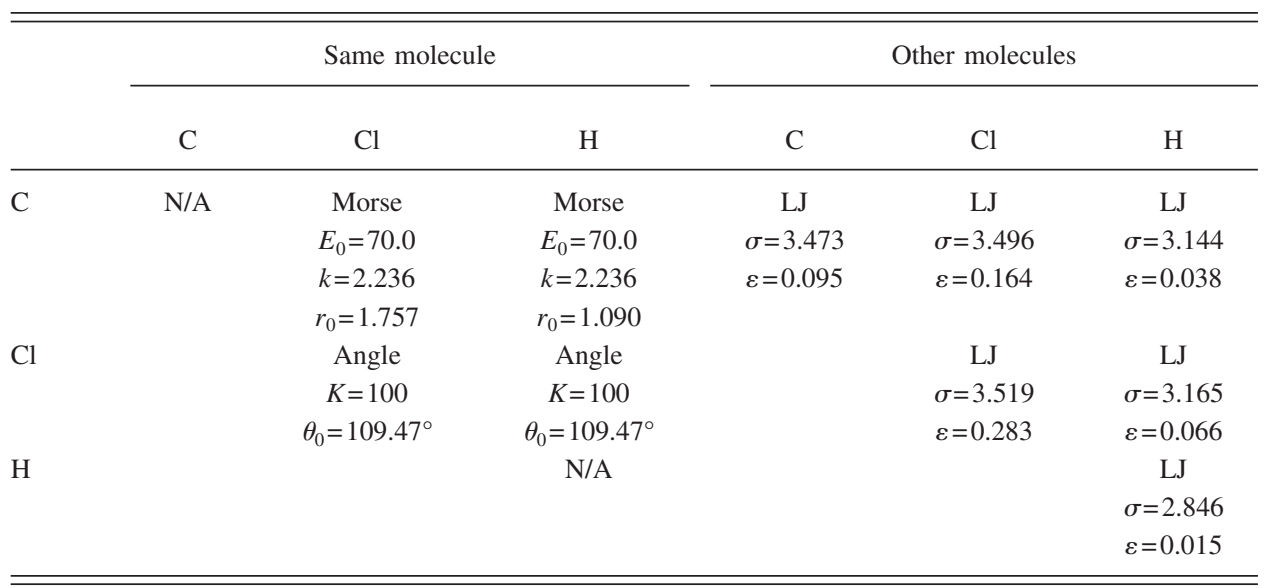

Data were sampled in an NVE (constant number, volume, and energy) run of $1 \times 10^{5}$ time steps with thermodynamic data acquired at every time step, while atomic positions and velocities were acquired every 25 time steps. The $N V E$ ensemble is used for the data acquisition because the Nosé-Hoover thermostat used for an NVT ensemble scales the atomic velocities and the Berendsen barostat used for an $N P T$ ensemble scales the cell volume, and consequently the bond lengths. Both the thermostat and the barostat thus interfere with a quantity that we wish to measure.

Repetition of the NPT-NVT-NVE cycle enables data to be obtained at different volumes (and hence pressures). In each case, the mean temperature and mean pressure were monitored during the $N V E$ runs to assess the state of equilibrium in each case. The mean temperature was close to $298 \mathrm{~K}$ and no pressure drift was observed, confirming that each system was properly equilibrated during the $N V T$ run.

\section{B. Simulation of chloroform vapor}

MD simulations of chloroform vapor at NTP are undertaken so that the bond lengths and positions of the Raman peaks can be compared to the liquid at NTP. The solvation pressure experienced by each liquid molecule is reflected by a shortening of the mean bond lengths compared to the vapor and can be observed directly. The change in bond length can then be compared to such changes due to the application of hydrostatic pressure to the liquid in simulations described in the previous section.

The vapor is modeled by placing a single chloroform molecule in a simulation cell and employing the NVE ensemble. The cell is sufficiently large that any interaction between the molecule and its images due to periodic boundary conditions is negligible. A larger number of time steps are required for a run, typically $10^{8}$, in order to extract data with good statistics. The temperature fluctuated by about $100 \mathrm{~K}$ during the simulations with the mean temperatures of each run lying in the range of $250-350 \mathrm{~K}$.

\section{The potentials}

The Dreiding potentials were used to describe the intraand intermolecular forces. ${ }^{14}$ This is a simple set of potentials with parameters that have been optimized against a broad range of properties for small molecules. We used the Morse potential for the intramolecular bonds, given by

$$
U=E_{0}\left[\left(1-e^{-k\left(r-r_{0}\right)}\right)^{2}-1\right],
$$

where $r$ is the separation between a pair of atoms and values of $E_{0}, k$, and $r_{0}$ are presented in Table I. Valence bond-angle potentials have the form

$$
U=\frac{1}{2} K\left(\theta_{i j k}-\theta_{0}\right)^{2},
$$

where $\theta_{i j k}$ is the angle subtended at atom $j$ by atoms $i$ and $k$ and the parameters $K$ and $\theta_{0}$ are again presented in Table I. The Lennard-Jones potential was used for intermolecular interactions, with

$$
U=4 \varepsilon\left[\left(\frac{\sigma}{r}\right)^{12}-\left(\frac{\sigma}{r}\right)^{6}\right],
$$

with values of $\varepsilon$ and $\sigma$ listed in Table I. Finally, the Coulomb potential was included, with Gasteiger ${ }^{15}$ charges for $\mathrm{C}$ of $+0.1801 e$, for $\mathrm{Cl}$ of $-0.0874 e$, and for $\mathrm{H}$ of $+0.0821 e$.

\section{RESULTS}

\section{A. Bulk properties of chloroform}

It is important to compare physical quantities for bulk chloroform obtained from the MD model with experimental values to assess the quality of the Dreiding potentials for chloroform. Results are summarized in Table II.

The density of chloroform at NTP is $1390 \mathrm{~kg} \mathrm{~m}^{-3}$, in reasonable agreement with the experimental value of $1480 \mathrm{~kg} \mathrm{~m}^{-3}$. The difference, about $6 \%$, is typical of a MD simulation of a liquid. The CED was calculated by using the difference of the configurational energy between the liquid and the vapor state. The configurational energy is the potential energy due to inter- and intramolecular forces. The CED is found to be 3.49 kbars which is in very good agreement with the experimental value of 3.61 kbars.

Previous MD simulations of hydrocarbons ${ }^{16,17}$ have shown a strong correlation between the CED and the internal pressure $\Pi_{i}$ where, 
TABLE II. Comparison of some experimental and MD values for chloroform. Note that the unit for the cohesive energy density is $1 \mathrm{kbar}=10^{5} \mathrm{~J} \mathrm{~m}^{-3}$.

\begin{tabular}{|c|c|c|c|}
\hline & & Experiment & MD \\
\hline Density $\left(\mathrm{kg} \mathrm{m}^{-3}\right)$ & & 1480 & $1390 \pm 10$ \\
\hline Cohesive energy density (kbar) & & 3.61 & $3.49 \pm 0.06$ \\
\hline Internal pressure $\Pi_{i}(\mathrm{kbar})$ & & 3.69 & $3.44 \pm 0.14$ \\
\hline Bulk modulus at 1 bar (GPa) & & 1 & $1.0 \pm 0.1$ \\
\hline Bulk modulus at $1 \mathrm{kbar}(\mathrm{GPa})$ & & 1.87 & $1.77 \pm 0.14$ \\
\hline $\mathrm{RDF} \mathrm{Cl}-\mathrm{Cl}(\AA)$ & & 3.85 & 3.8 \\
\hline RFD Cl-H ( $(\AA)$ & & 3 & 3.3 \\
\hline \multirow[t]{2}{*}{$251 \mathrm{~cm}^{-1}$ mode } & $\alpha\left(\mathrm{kbar}^{-1}\right)$ & 0.74 & 0.81 \\
\hline & $\beta\left(\mathrm{kbar}^{-2}\right)$ & -0.021 & -0.025 \\
\hline \multirow[t]{2}{*}{$368 \mathrm{~cm}^{-1}$ mode } & $\alpha\left(\mathrm{kbar}^{-1}\right)$ & 0.37 & 0.70 \\
\hline & $\beta\left(\mathrm{kbar}^{-2}\right)$ & -0.008 & -0.018 \\
\hline
\end{tabular}

$$
\Pi_{i}=\left(\frac{\partial U}{\partial V}\right)_{T} .
$$

For most polymers $\Pi_{i}$ is used since it is impossible to measure the heat of vaporization. For the calculation of the internal pressure $\Pi_{i}$, only the configurational energy was used. The kinetic energy is a function of $T$ only and can therefore be ignored. The configurational energy was plotted as a function of volume for pressures less than $6.8 \mathrm{kbars}$ and fitted with a straight line. The slope, $\Pi_{i}$, was found to be 3.44 kbars, very close to the value of the CED. This comes as no surprise as the equivalence of CED and $\Pi_{i}$ is well known for molecules of spherical symmetry which interact only through dispersive forces. ${ }^{18,19}$

The bulk modulus was found at two pressures by fitting the pressure and volume data to the Murnaghan equation of state,

$$
P=\frac{B_{0}}{B^{\prime}}\left(\left(\frac{V_{0}}{V(P)}\right)^{B^{\prime}}-1\right)
$$

where $B$ is the bulk modulus, the subscript 0 refers to NTP, and $B^{\prime}$ is the pressure coefficient of the bulk modulus. ${ }^{9}$ Once again, the agreement between simulation and experiment is very satisfactory.

The radial distribution functions (RDFs) of the liquid are obtained at NTP and the first peak position for the $\mathrm{Cl}-\mathrm{Cl}$ and $\mathrm{Cl}-\mathrm{H}$ RDFs are presented in Table II. The simulation slightly overestimates the $\mathrm{Cl}-\mathrm{H}$ peak position. Overall, the agreement is as good as can be expected using the generic Dreiding potentials.

\section{B. Bond lengths}

In Fig. 1 the mean $\mathrm{C}-\mathrm{Cl}$ and $\mathrm{C}-\mathrm{H}$ bond lengths obtained from the MD simulations are plotted against the pressure for the chloroform liquid. Consistent with the SPM, we have adopted the practice of plotting the results of the liquid simulation at NTP at 0 kbar. Thus the bond lengths in the vapor phase, which are also shown, are plotted at the negative pressure corresponding to the simulated CED. Strictly, in the SPM, the molecules are under zero total pressure in the gas phase and under the solvation pressure in the liquid at NTP.
However, it is convenient to place the zero of the pressure axis for the liquid at NTP and we do this in all the relevant figures below.

The mean $\mathrm{C}-\mathrm{Cl}$ and $\mathrm{C}-\mathrm{H}$ bond lengths in the chloroform liquid at NTP are shorter than for the vapor, clearly demonstrating the solvation pressure effect. The $\mathrm{C}-\mathrm{Cl}$ bond length appears to respond to solvation pressure in the same manner as applied hydrostatic pressure, since the bond length in the vapor lies, within error, on the trend line of the high-pressure data. The $\mathrm{C}-\mathrm{H}$ bond does not agree so well, but still lies near the trend line. The uncertainty in the mean $\mathrm{C}-\mathrm{H}$ bond length is large in the vapor phase due to the larger vibrations associated with its small mass of $\mathrm{H}$. The $\mathrm{C}-\mathrm{H}$ bond shortens slightly less under applied hydrostatic pressure then is consistent with the shortening from vapor to liquid due to solvation pressure, but later results show that it is the hydrogen atom that is implicated in the masking interaction.

\section{Pressure dependence of the Raman frequencies}

The vibrational spectrum is obtained as the power spectrum of the ensemble, defined as the Fourier transform of the velocity autocorrelation function. The sampling period of 25 time steps (5 fs) is less than half the period of the highest vibrational frequency expected to be present $\left(3000 \mathrm{~cm}^{-1}\right)$, so avoiding aliasing.

The vibrational spectrum of the model is compared with the Raman spectrum of liquid chloroform in Fig. 2. The peaks obtained from the MD simulations correspond to experimental peaks with discrepancies of up to $100 \mathrm{~cm}^{-1}$ to be expected. Our simulation has four Raman peaks with dis-
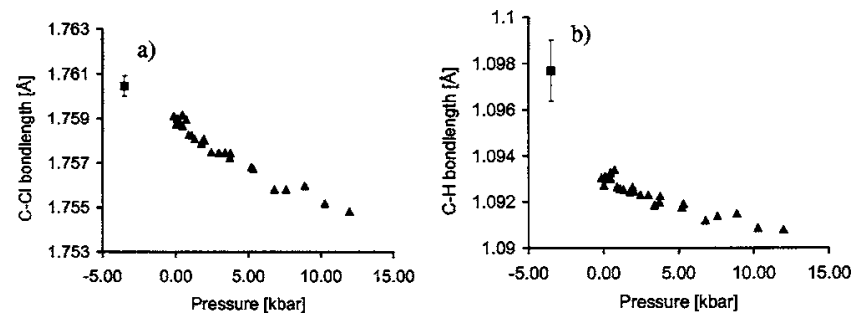

FIG. 1. Simulated bond lengths for the chloroform molecule in the liquid phase as a function of pressure $(\mathbf{\Delta})$. The bond lengths of an isolated molecule (ם) are plotted at -3.49 kbars. 


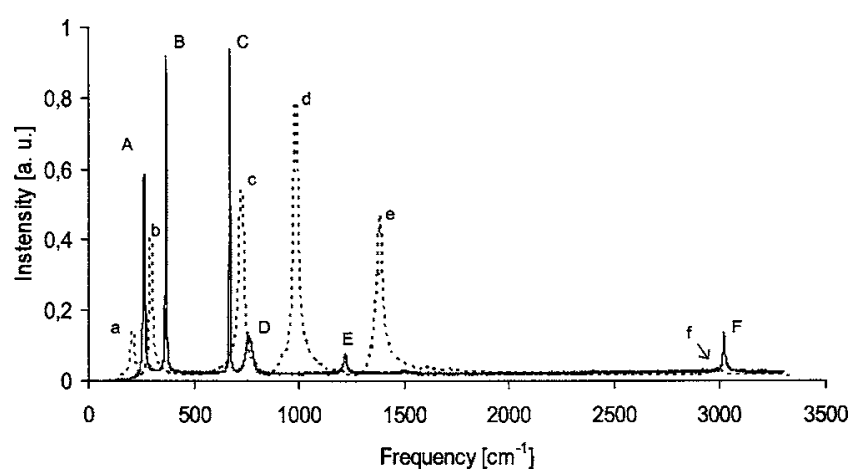

FIG. 2. Experimental Raman spectrum of chloroform liquid at ambient pressure (solid line) and the power spectrum as obtained from the MD (dotted line). The peaks are labeled as follows (uppercase for experimental, lowercase for simulated peaks): $\mathrm{A}$, a: antisymmetric $\mathrm{C}-\mathrm{Cl}$ bending mode; $\mathrm{B}, \mathrm{b}$ : symmetric $\mathrm{C}-\mathrm{Cl}$ bending mode; $\mathrm{C}$, c: symmetric $\mathrm{C}-\mathrm{Cl}$ stretch; $\mathrm{D}$, d: antisymmetric $\mathrm{C}-\mathrm{Cl}$ stretch; $\mathrm{E}$, e: $\mathrm{C}-\mathrm{H}$ bending mode; and $\mathrm{F}, \mathrm{f}: \mathrm{C}-\mathrm{H}$ stretch.

crepancies less than $100 \mathrm{~cm}^{-1}$ and two $(\mathrm{C}-\mathrm{H}$ bending and $\mathrm{C}-\mathrm{Cl}$ antisymmetric stretch) with larger discrepancies. This is adequate for our purposes. In the figures below, the simulated peaks are shifted to coincide with the experimental peaks at the zero of the pressure axis. Since it is only the shifts with pressure and solvation that matter, this is equivalent to plotting the frequency shifts $\Delta \nu$.

Figures 3-5 show the frequencies of the six strong peaks in the chloroform spectrum of Fig. 2 plotted as a function of pressure, together with the corresponding peaks in the vibrational spectrum of the MD simulation. The frequencies in the vapor phase are also shown, plotted at the negative pressure corresponding to the simulated CED. For the $261 \mathrm{~cm}^{-1}$ antisymmetric $\mathrm{C}-\mathrm{Cl}$ bending mode [Fig. 3(a)], agreement between MD and experiment is excellent. Quadratic fits to the experimental and MD pressure data were made (the fit parameters are in Table II) and the MD fit is plotted on the figure. Note that the MD vapor point falls very close to the fit, extrapolated to negative pressure. This peak was not observed in the experiment in the vapor. This result is as predicted by the SPM.

For the $368 \mathrm{~cm}^{-1}$ symmetric $\mathrm{C}-\mathrm{Cl}$ bending mode [Fig. $3(\mathrm{~b})$ ], the agreement between experiment and MD is reasonable. Agreement could be improved by using a more accurate value of $K$, the parameter defining the valence bond-angle potential. However, the important result is that both the experimental and MD vapor points fall very close to their respective quadratic fits to the pressure data. That is, both experiment and MD agree well with the predictions of the SPM.

Figure 4 shows the Raman peak position of the $667 \mathrm{~cm}^{-1}$ symmetric and the $757 \mathrm{~cm}^{-1}$ antisymmetric $\mathrm{C}-\mathrm{Cl}$ modes for the vapor and for the liquid as a function of pressure. Once again, the MD vapor points lie very close to the quadratic fits to the MD pressure data as predicted by the SPM. The experimental data show that the peak position increases with pressure but to a much lesser extent than predicted by the MD simulations. It is clear that the Dreiding potentials omit some terms that soften these specific modes when the bond lengths are shortened. Experimentally, these modes are soft-
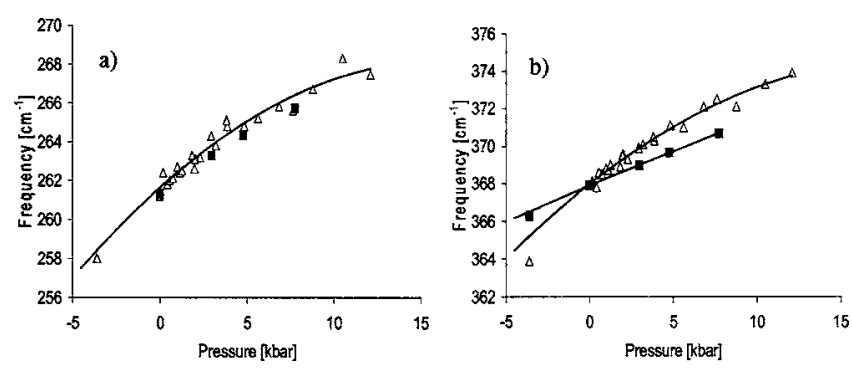

FIG. 3. Frequency of the antisymmetric $\mathrm{C}-\mathrm{Cl}$ bending (a) and symmetric $\mathrm{C}-\mathrm{Cl}$ bending (b) modes as a function of pressure, calculated in the $\mathrm{MD}(\triangle)$ and measured experimentally $(\boldsymbol{\square})$. The solid lines represent quadratic fits to the liquid data only. In (b) a fit has also been performed on the experimental data. MD values are shifted vertically by $54 \mathrm{~cm}^{-1}$ (a) and $73 \mathrm{~cm}^{-1}$ (b).

ened in the liquid compared to the vapor, resulting in peak positions for the vapor that do not lie on the trend lines for the liquid under pressure.

For the $1216 \mathrm{~cm}^{-1} \mathrm{C}-\mathrm{H}$ bending mode and the $3020 \mathrm{~cm}^{-1} \mathrm{C}-\mathrm{H}$ stretching mode (Fig. 5), all the vapor points, both experimental and MD, lie off the quadratic fits. The vibrations of the $\mathrm{H}$ atom, with its small mass, are most likely to be affected by the proximity of neighboring molecules in the liquid. For example, the increasing proximity with pressure of three negatively charged $\mathrm{Cl}$ atoms to the $\mathrm{H}$ atom on a neighboring molecule will affect the vibrational properties of the $\mathrm{H}$ atom superposed on the change associated with the shortening of bonds due to the solvation pressure. This confirms the results from Sec. III B in relation to the $\mathrm{C}-\mathrm{H}$ bond lengths obtained from simulation. The experimental results in Fig. 5 also show no significant change of the peak positions with pressure. Both sets of results confirm the presence of a masking interaction in real chloroform liquid that affects bond lengths and Raman frequencies, softening its modes with pressure.

The experimental results for the $667 \mathrm{~cm}^{-1}$ symmetric mode, the $757 \mathrm{~cm}^{-1}$ antisymmetric $\mathrm{C}-\mathrm{Cl}$ mode (Fig. 4), the $1216 \mathrm{~cm}^{-1} \mathrm{C}-\mathrm{H}$ bending mode, and the $3020 \mathrm{~cm}^{-1} \mathrm{C}-\mathrm{H}$ stretching mode (Fig. 5) are almost independent of pressure, whereas the MD simulations reveal an increase in frequency in all cases. We believe that, as molecules are pushed together by the applied pressure in the real system, the $\mathrm{C}-\mathrm{H}$ or $\mathrm{H}-\mathrm{H}$ interatomic potentials are modified to annul the frequency changes predicted by the MD simulations in which the potentials are density independent. For example, three
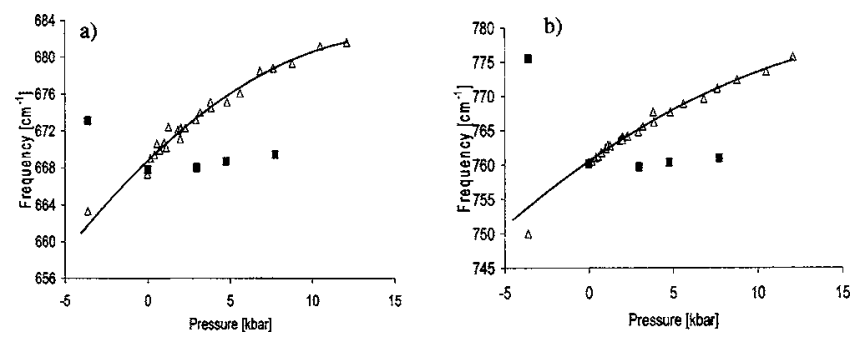

FIG. 4. Frequency of the symmetric $\mathrm{C}-\mathrm{Cl}$ stretch (a) and antisymmetric $\mathrm{C}-\mathrm{Cl}$ stretch (b) modes as a function of pressure, calculated in the MD $(\triangle)$ and measured experimentally $(\boldsymbol{\square})$. The solid lines represent quadratic fits to the liquid data only. MD values are shifted vertically by $-57 \mathrm{~cm}^{-1}$ (a) and $-222 \mathrm{~cm}^{-1}$ (b). 

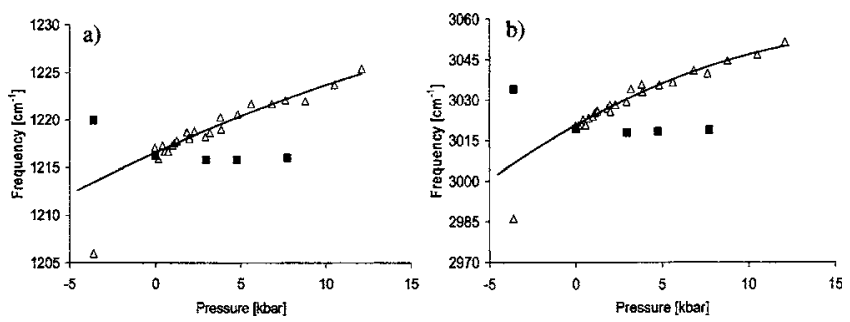

FIG. 5. Frequency of the $\mathrm{C}-\mathrm{H}$ bending (a) and $\mathrm{C}-\mathrm{H}$ stretch (b) modes as a function of pressure, calculated in the MD $(\triangle)$ and measured experimentally (ם). The solid lines represent quadratic fits to the liquid data only. MD values are shifted vertically by $-164 \mathrm{~cm}^{-1}$ (a) and $37 \mathrm{~cm}^{-1}$ (b).

negatively charged $\mathrm{Cl}$ atoms on one molecule might induce a density-dependent increased effective positive partial charge on a $\mathrm{H}$ atom in a neighboring molecule.

\section{DISCUSSION AND CONCLUSIONS}

The generic Dreiding potential set provides a good approximation to the interatomic potentials in chloroform with most bulk properties giving satisfactory agreement with experiment. Ab initio modeling may provide a route to improved interatomic potentials that could better replicate the ambient-pressure vibrational frequencies. However, that is not the objective of the work reported here.

There are several intriguing points raised by the present results. First, the Dreiding potentials yield better agreement with the model of solvation pressure than does the real chloroform. The difference in bond lengths between the MD simulations of vapor and liquid at NTP clearly demonstrates the solvation pressure effect in the liquid. Moreover, the pressure dependence of the bond lengths and vibrational frequencies shows that the magnitude of the solvation pressure is equal to the CED, with the exception of $\mathrm{C}-\mathrm{H}$ bond data. Here, the small mass of the $\mathrm{H}$ means that the bond length and vibrational behavior are vulnerable to the local orientation of the molecules in the liquid. We note that a similar observation was made with ethanol. ${ }^{11}$

It follows that the SPM is general, but that specific interactions occur in the real system so as to mask the solvation pressure effects. The experimental results for the antisymmetric $\mathrm{C}-\mathrm{Cl}$ bending and symmetric $\mathrm{C}-\mathrm{Cl}$ bending mode as a function of pressure are also in good agreement with the SPM.

However, because experiment agrees less well with the solvation pressure model than does the MD simulation, the Dreiding potentials must omit an interaction or a perturbation of importance to the pressure or density dependence of the vibrational spectrum and, specifically, to those peaks in it in which the $\mathrm{CH}$ group is involved. It is not a large term that is omitted, for the softening that it causes amounts to no more than $1 \%-3 \%$ over the density range from $\rho=0$ in the vapor to $\rho=2 \mathrm{~g} \mathrm{~cm}^{-3}$ at the highest pressures. The obvious candidate for the missing term is a perturbation of the hydrogen two- and three-body intramolecular potentials in the presence of other molecules. Such a perturbation need only soften the potentials for the modes in which the carbon and hydrogen move (or, equivalently, increase the reduced mass of these modes); it would have only an insignificant effect on the CED of the liquid. Indeed, the experimental determination of the pressure dependence of Raman modes together with MD simulation may provide a tool to examine such effects. These effects may also be considered as solventsolute interactions, although the solute here is the same molecular type as the solvent, and we would expect the statistical mechanical descriptions discussed in Sec. I (Refs. 2 and 5-7) to account for these masking interactions. Indeed, the general form of the Raman shift calculated by MeléndézPagán and Ben-Amotz using the statistical mechanical description is a linear dependence on density plus a linear dependence on pressure. ${ }^{2}$ The differences between the MD results and the experimental data in Figs. 4 and 5 can be accurately fitted to this general form.

Finally, it is noted that the present approach to solvation effects could not usefully be extended to lower fluid densities. Indeed, it would be unphysical to attribute a cohesive energy density and consequent solvation pressure to a fluid at a reduced density where phase separation into liquid and gas takes place. It would be interesting, however, to consider low-density fluids at high temperatures where the reduced density is stable.

In conclusion, comparisons of experiment and MD confirm that it is masking effects, or solvent-solute interactions, that vitiate purely experimental demonstrations of the validity of the solvation pressure model. The key result here is that a simplification of the true potentials can eliminate or reduce masking effects, thereby exhibiting more clearly the effect of the solvation pressure. These MD simulations of chloroform expose the ubiquitous nature of solvation pressure.

\section{ACKNOWLEDGMENTS}

The authors would like to thank Queen Mary, University of London and the Engineering and Physical Sciences Research Council (EPSRC), UK for their support of this project.

${ }^{1}$ E. Fisher and H. G. Drickamer, J. Chem. Phys. 24, 548 (1956) and references therein.

${ }^{2}$ Y. Meléndéz-Pagán and D. Ben-Amotz, J. Phys. Chem. B 104, 7858 (2000).

${ }^{3}$ L. Onsager, J. Am. Chem. Soc. 58, 1486 (1936).

${ }^{4}$ E. Bauer and M. Magat, J. Phys. Radium 9, 319 (1938).

${ }^{5}$ A. D. Buckingham, G. B. B. Sutherland, J. A. Pople, and A. D. E. Pullin,Proc. R. Soc. London, Ser. A 255, 32 (1960).

${ }^{6}$ K. S. Schweizer and D. Chandler, J. Chem. Phys. 76, 2296 (1982).

${ }^{7}$ M. R. Zakin and D. R. Herschbach, J. Chem. Phys. 89, 2380 (1988).

${ }^{8}$ E. A. Grulke, Polymer Handbook, 4th ed. (Wiley, New York, 1998).

${ }^{9}$ CRC Handbook of Chemistry and Physics, 58th ed. (CRC, Boca Raton, FL, 1978).

${ }^{10}$ J. R. Wood, M. D. Frogley, E. R. Meurs, A. D. Prins, T. Peijs, D. J. Dunstan, and H. D. Wagner, J. Phys. Chem. B 103, 10388 (1999).

${ }^{11}$ N. W. A. van Uden, H. Hubel, D. A. Faux, A. C. Tanczos, B. Howlin, and D. J. Dunstan, J. Phys.: Condens. Matter 15, 1577 (2003).

${ }^{12}$ N. W. A. van Uden, H. Hubel, D. A. Faux, D. J. Dunstan, and C. Royer, High Press. Res. 23, 205 (2003). 
${ }^{13}$ W. Smith and T. R. Forester, J. Mol. Graphics 14, 136 (1996).

${ }^{14}$ S. L. Mayo, B. D. Olafson, and W. A. Goddard III, J. Phys. Chem. 94, 8897 (1990).

${ }^{15}$ J. Gasteiger and M. Marsili, Tetrahedron 36, 3219 (1980).

${ }^{16}$ J. K. Maranas, M. Mondello, G. S. Grest, S. K. Kumar, P. G. Debenedetti, and W. W. Graessley, Macromolecules 31, 6991 (1998).

${ }^{17}$ A. Indrakanti, J. K. Maranas, and S. K. Kumar, Macromolecules 33, 8865 (2000).

${ }^{18}$ J. H. Hildebrand, Phys. Rev. 34, 984 (1929).

${ }^{19}$ G. Allen, G. Gee, and G. J. Wilson, Polymer 1, 456 (1960). 